\title{
Diagnóstico de la calidad ambiental del río Oja (La Rioja, España) mediante el análisis de la comunidad de macroinvertebrados bentónicos
}

\author{
Martínez-Bastida J.J., ${ }^{1, *}$ Arauzo M. ${ }^{1}$, Valladolid M. ${ }^{2}$ \\ 1 Departamento de Contaminación Ambiental. Centro de Ciencias Medioambientales, CSIC. Serrano 115, 28006 \\ Madrid. \\ 2 Departamento de Biodiversidad y Biología Evolutiva. Museo Nacional de Ciencias Naturales, CSIC. José Gu- \\ tiérrez Abascal 2, 28006 Madrid.
}

* Autor responsable de la correspondencia: Martinez.Bastida@ccma.csic.es

\begin{abstract}
RESUMEN
Diagnóstico de la calidad ambiental del río Oja (La Rioja, España) mediante el análisis de la comunidad de macroinvertebrados bentónicos

En la cuenca del río Oja confluyen una serie de factores de origen antrópico que pueden afectar al equilibrio natural del ecosistema fluvial, tales como el desarrollo de una intensa actividad agrícola de regadío y la descarga de efluentes procedentes de pequeños núcleos urbanos e industriales. En mayo de 2003 se realizó una campaña de estudio del río Oja. Se presenta una valoración de la calidad de sus aguas mediante la aplicación de índices bióticos y el análisis de la estructura de las comunidades macrobentónicas. El índice IBMWP alcanzó valores elevados en todas las estaciones, indicando un buen estado de conservación, aunque con tendencia a disminuir aguas abajo con respecto a la cabecera. Los índices de EPT riqueza, EPT/Chironomidae y Shannon presentaron valores reducidos en las estaciones del tramo medio-bajo del río.

En el análisis multivariante se aprecian dos factores que explican la estructura de la comunidad macrobentónica del río: el primero define un gradiente longitudinal de contaminación (orgánica y por nitrato), temperatura, $\mathrm{pH}$ y salinidad, y el segundo recoge las variaciones de caudal y el aporte de fosfato. La intensidad con que se manifiestan los impactos sobre la calidad del sistema es reducida y de carácter puntual, si bien existe el riesgo de que se acentúe durante el periodo estival.
\end{abstract}

Palabras clave: Macroinvertebrados, Río Oja, IBMWP, calidad del agua, factores antropogénicos.

\begin{abstract}
Environmental quality diagnosis in the Oja river (La Rioja, Spain) through the analysis of the benthonic macroinvertebrate community

In the Oja River basin, a series of factors of anthropic origin act together, that can affect the natural fluvial ecosystem equilibrium, such as intensive agricultural irrigation and sewage effluent discharges from small urban and industrial centres. In May 2003 a study on the Oja River was performed. An evaluation of the quality of its waters is presented, from several biological indices as well as the analysis of the benthonic macroinvertebrate community structure. The IBMWP biological index presented high scores at every station showing a good quality, although decreasing downstream. The EPT richness, EPT/Chironomidae ratio and Shannon's diversity indices showed low scores in the medium and final stretches of the river. Multivariate analysis shows two factors that explain the macroinvertebrate community structure: the first one defines a longitudinal gradient of organic and nitrate pollution, temperature, $\mathrm{pH}$ and salinity, and the second one includes flow variations and orthophosphate inputs. The intensity of the impacts over the ecosystem is punctual, even though the risk exists that it may become more important during the summer.
\end{abstract}

Keywords: Macroinvertebrates, Oja River, IBMWP, water quality, anthropogenic factors. 


\section{INTRODUCCIÓN}

La calidad del agua, la disponibilidad de alimento y las características del sustrato, son los principales factores que gobiernan la abundancia y distribución del bentos. Por este motivo, la relación entre la macrofauna bentónica y los factores abióticos es una herramienta ampliamente utilizada para la valoración del estado de calidad de gran parte de las redes fluviales (Rodríguez et al., 1994). De hecho, la Directiva Marco del Agua 2000/60/CE establece que los indicadores biológicos han de ser los que determinen en última instancia el estado de una masa de agua. El índice BMWP' (Alba-Tercedor \& Sánchez Ortega, 1988; Alba-Tercedor, 1996) ha sido uno de los índices bióticos más empleados (Rico et al., 1992), si bien debe considerarse como una propuesta abierta, debido a la todavía limitada información taxonómica y ecológica sobre la macrofauna fluvial mediterránea (Vivas et al., 2002). Una versión actualizada de este índice es el IBMWP (Iberian Biomonitoring Working Party: Alba-Tercedor et al., 2002). Otros índices bióticos comúnmente empleados en la evaluación del estado ecológico de los ríos son el IASPT (Armitage et al., 1983), el índice EPT (Barbour et al., 1999, basado en la riqueza de los órdenes efemerópteros, plecópteros y tricópteros) y el índice EPT/Chironomidae (Barbour et al., 1999, basado en la abundancia). Estos índices, sin embargo, presentan una marcada tendencia a la penalización de los tramos fluviales más térmicos y mineralizados conduciendo, en ocasiones, a interpretaciones erróneas en las que se asocian procesos naturales con pérdida de calidad (Vivas et al., 2002). Por este motivo, suelen ser complementados con el uso de otros parámetros que describen la estructura de la comunidad: riqueza taxonómica, densidad, abundancia relativa e índices de diversidad. Los índices de diversidad constituyen una herramienta útil para condensar información que puede resultar compleja, y facilitar la interpretación (Guerold, 2000). El índice de Shannon \& Weaver (1949) es uno de los más utilizados. La interpretación debe realizarse, no obstante, con cierta cautela, ya que el nivel taxonómico empleado para el cálculo de los mismos puede in- fluir en el resultado, tendiendo a subestimarse el grado de alteración real que soporta la comunidad cuando se utilizan niveles de familia u orden (Guerold, 2000). Estos índices no permiten realizar interpretaciones ecológicas más profundas (Guerold, 2000). Las técnicas de análisis multivariante proporcionan, sin embargo, una herramienta complementaria que permite explorar las relaciones entre la distribución de los taxones de la comunidad y las variables ambientales.

En este estudio, se ha hecho uso del índice IBMWP para diagnosticar la calidad ambiental

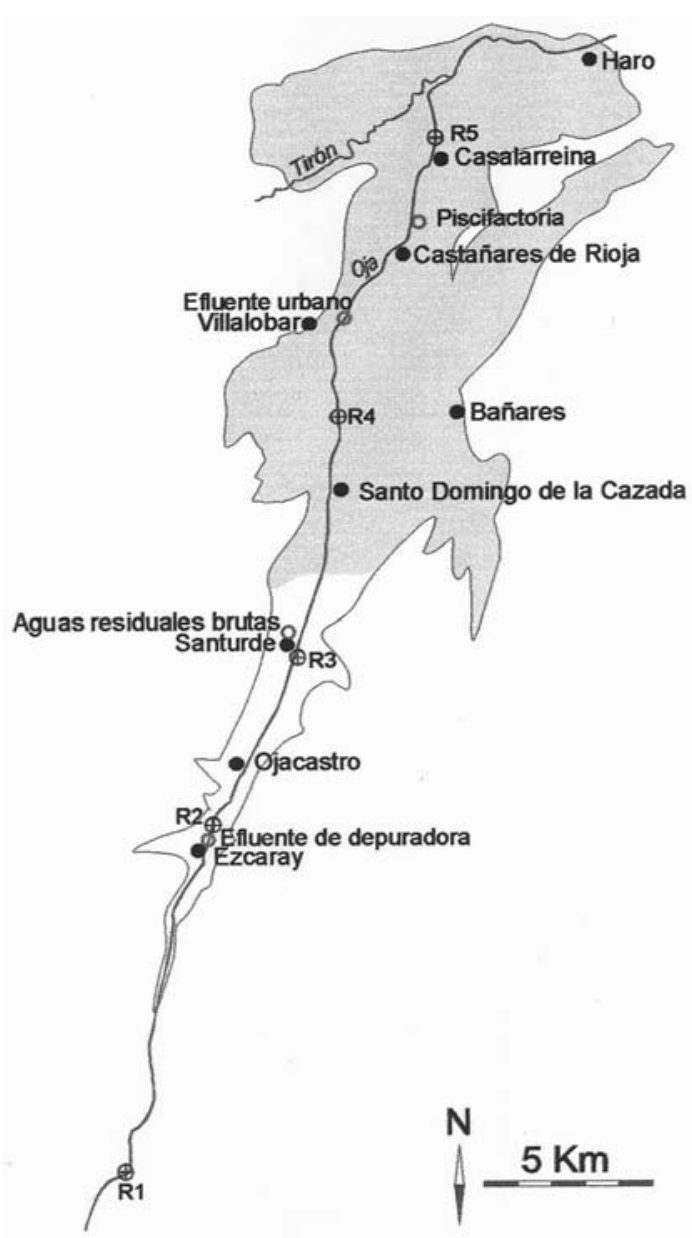

Figura 1. Mapa de la Unidad Hidrogeológica 09-04-03. Situación del río Oja, de las estaciones de muestreo y de los puntos de impacto que afectan al sistema. En gris: agricultura de regadío. Map of the Hydrogeological Unit 09-04-03. Location of the Oja River, the sampling stations and the impact points that affect the system. In grey: irrigation agriculture. 
Tabla 1. Frecuencias relativas (\%) de los grupos taxonómicos de la comunidad macrobentónica en cada estación de muestreo. Relative frequency (\%) of the macroinvertebrate community taxonomic groups at each sampling station.

\begin{tabular}{lccccc}
\hline & Cabecera & Ezcaray & Santurde & Santo Domingo & Casalarreina \\
\hline Ephemeroptera & 28.17 & 12.03 & 13.53 & 25.69 & 4.45 \\
Plecoptera & 4.96 & 8.42 & 4.74 & 0.70 & 0.25 \\
Trichoptera & 6.44 & 0.75 & 0 & 0 & 0.06 \\
Coleoptera & 8.33 & 1.05 & 0.26 & 0.11 & 0.20 \\
Diptera & 24.11 & 24.66 & 76.88 & 65.97 & 87.94 \\
Chironomidae & 22.22 & 22.71 & 75.63 & 60.45 & 83.93 \\
Oligochaeta & 12.30 & 43 & 1.34 & 6.38 & 2.54 \\
Amphipoda & 0 & 4.06 & 0.18 & 0 & 3.62 \\
Isopoda & 0 & 1.65 & 0.54 & 0 & 0.05 \\
Ostracoda & 4.07 & 0.90 & 0.36 & 0.08 & 0.14 \\
Copepoda & 0.20 & 0.60 & 0.36 & 0.43 & 0.34 \\
Turbellaria & 0.39 & 0 & 0 & 0 & 0 \\
Hirudinea & 0 & 0.30 & 0.36 & 0 & 0 \\
Nematoda & 0 & 0 & 0 & 0.22 & 0 \\
Gasteropoda & 0 & 0.45 & 0 & 0 & 0.04 \\
\hline
\end{tabular}

del río Oja (La Rioja, España). Con la aplicación del mismo, se pretende obtener información sobre el grado de alteración en que se encuentran los distintos tramos del río. Los índices EPT (riqueza de familias) y EPT/Chironimidae (abundancia) han sido empleados con el mismo fin, como información complementaria, puesto que son índices de tipo cuantitativo, ofrecen una visión rápida del estado del sistema y muestran algunas características de la comunidad (riqueza de taxones indicadores y relaciones de dominancia entre algunos de los principales órdenes del sistema). Se ha estudiado, asimismo, la evolución de la estructura de la comunidad a lo largo del gradiente longitudinal del río, mediante el uso de índices de abundancia relativa, riqueza, densidad y diversidad de Shannon-Weaver. Por último, se ha analizado la relación entre los factores abióticos y la estructura de la comunidad macrobentónica del río, mediante el uso de técnicas multivariantes con el fin de identificar los principales factores ambientales que influyen sobre la comunidad.

\section{MATERIAL Y MÉTODOS}

\section{Área de estudio}

El río Oja (La Rioja, España) nace en la Sierra de la Demanda (Ezcaray), a 1880 metros de altitud, y desemboca en el Tirón, a 480 metros (pertenecientes ambos a la cuenca del Ebro). Tiene una longitud de $47 \mathrm{~km}$ y una pendiente media del $29 \%$ (Zaldívar, 1994), lo que favorece un flujo rápido del agua. El sustrato geológico sobre el que discurre el río y su acuífero aluvial (perteneciente a la Unidad Hidrogeológica 0904-03), lo conforman depósitos del Cuaternario (coluviales, aluviales, terrazas aluviales, terrazas bajas y conos de pie de monte), con una litología de gravas gruesas aluviales, gravas polígénicas, arenas y limos (Instituto Geológico y Minero de España, 1985). El régimen hidrológico es de tipo pluvionival. La Unidad Hidrogeológica ocupa una superficie aproximada de $148.5 \mathrm{~km}^{2}$.

El río Oja recibe, a lo largo de su curso, algunas descargas de efluentes residuales procedentes de pequeños núcleos urbanos e industriales (Fig. 1). Tal es el caso de la estación depuradora de Ezcaray, que trata agua residual de tipo mixto, y vierte su efluente sobre el río. Las aguas residuales brutas de Santurde se infiltran directamente en el acuífero y las de Villalobar se incorporan al río a través de uno de un arroyo tributario. En las proximidades de Castañares existe una piscifactoría. Por otra parte, la zona media y baja de la cuenca está destinada a la agricultura de regadío, que favorece la lixiviación de los compuestos nitrogenados empleados como fertilizantes y su trans- 
porte hacia las masas de agua. El agua de riego se obtiene, principalmente, de la explotación del acuífero aluvial, lo que propicia que el nivel freático experimente grandes oscilaciones a lo largo del año, llegando incluso a desaparecer el agua del cauce durante el periodo estival. Debido a la irregularidad del caudal, existe riesgo de crecidas, generalmente durante la primavera. A principios del mes de mayo del 2003, semanas antes del muestreo, las fuertes lluvias provocaron el desbordamiento de las aguas, inundando tierras de cultivo y carreteras.

\section{Metodología}

La campaña de muestreos se llevó a cabo durante la primavera del año 2003. Se establecieron cinco estaciones de muestreo a lo largo del recorrido del río, tendiendo en cuenta los puntos que eran objeto de algún tipo de perturbación conocida, a excepción de la primera estación (R1), situada en cabecera. La segunda estación (R2), se localizó a unos cien metros aguas abajo del vertido del efluente residual procedente de la depuradora de Ezcaray. El resto de las estaciones se ubicaron en Santurde (R3), dos kilómetros más abajo de Santo Domingo de la Calzada (R4) y en Casalarreina (R5, próxima a su confluencia con el Tirón), áreas de intensa actividad agrícola (Fig. 1). En cada estación se realizaron mediciones in situ de temperatura del agua, $\mathrm{pH}$, potencial redox (ORP), conductividad eléctrica y porcentaje de saturación de oxígeno (\% DO), mediante un sistema multiparámetrico portátil conectado a multisonda y kit pH/re-dox (ORP), (modelo YSI 556). Asimismo, se recogieron muestras simples para la determinación de la demanda química de oxígeno (DQO) y las concentraciones de nitratos y fosfatos. La DQO se determinó mediante la técnica del dicromato potásico (APHA, 1998). Los aniones se determinaron mediante cromatografía iónica (IC). El caudal se estimó como el producto del área de la sección transversal del río y la velocidad de la corriente (correntímetro).

El muestreo de los macroinvertebrados se realizó mediante el uso de un Surber $30 \times 30$ cm (250 $\mu \mathrm{m}$ de luz de malla), y una red de deriva, situada a contracorriente durante un periodo de 30 minutos. Se tomaron un mínimo de 6 y un máximo de 12 muestras en cada estación dependiendo de la anchura del río (más la de la red de deriva), distribuidas a lo largo de la sección transversal del cauce. Cuando la heterogeneidad ambiental de la sección requería incorporar alguna más, así se hacía. Las muestras de macroinvertebrados fueron conservadas inicialmente en formol y posteriormente separadas y determinadas hasta el nivel taxonómico de familia, mediante el uso de claves generales (p.e. Tachet et al., 2002).

La evaluación de la calidad biológica del sistema fluvial se realizó mediante la aplicación del índice IBMWP (Alba-Tercedor et al., 2002) en cada una de las estaciones muestreadas. También se hizo uso del índice EPT riqueza (basado en familias de efemerópteros, plecópteros y tricópteros) y el índice EPT/Chironomidae (basado en abundancia), como información complementaria. Para el análisis de la estructura de la comunidad se determinaron los parámetros de abundancia relativa, densidad (individuos $/ \mathrm{m}^{2}$ ), riqueza y el índice de diversidad de Shannon \& Weaver $\left(H^{\prime}\right)$ a partir de las familias. Por último, se utilizó el análisis de componentes principales (ACP) y el análisis de correspondencias (AC) del programa Statistica 6.0 (StatSoft, 2003), para estudiar la relación entre las variables ambientales y la estructura de la comunidad macrobentónica. El análisis de componentes principales se realizó a partir de la matriz formada por las siguientes variables: $\mathrm{pH}$, temperatura, caudal, porcentaje de saturación de oxígeno, potencial redox, conductividad, DQO, nitratos, fosfatos e índices IBMWP y $H^{\prime}$. Se realizó una normalización de las variables previa al análisis, mediante transformación logarítmica. El análisis de correspondencias, se realizó a partir de la matriz de abundancias de las familias de macroinvertebrados. En el diagrama de ordenación, sólo se representaron los taxones que alcanzaron valores significativos.

\section{RESULTADOS}

Se han identificado un total de 18 órdenes y 53 familias de macroinvertebrados acuáticos (más información Valladolid et al., 2006). Los gru- 
Tabla 2. Valores de IBMWP, rango de calidad, estado ecológico, nivel de calidad y colores asociados en cada estación de muestreo. IBMWP values, quality range, ecological state, quality level and associated colours at each station.

\begin{tabular}{lccccc}
\hline Estaciones & IBMWP & Rango & Estado ecológico & Calidad & Color \\
\hline Cabecera & 197 & $>150$ & Muy bueno & Aguas muy limpias & Azul \\
Ezcaray & 135 & $150-120$ & Bueno & Aguas limpias & Azul \\
Santurde & 118 & $101-120$ & Bueno & Aguas no contaminadas o alteradas de modo sensible & Azul \\
Santo Domingo & 106 & $101-120$ & Bueno & Aguas no contaminadas o alteradas de modo sensible & Azul \\
Casalarreina & 99 & $61-100$ & Aceptable & Son evidentes algunos efectos de la contaminación & Verde \\
\hline
\end{tabular}

pos con mayor abundancia y frecuencia de aparición fueron dípteros, seguido de efemerópteros (Tabla 1). Desde el punto de vista taxonómico, los órdenes mejor representados fueron nuevamente dípteros, con 14 familias, y tricópteros,
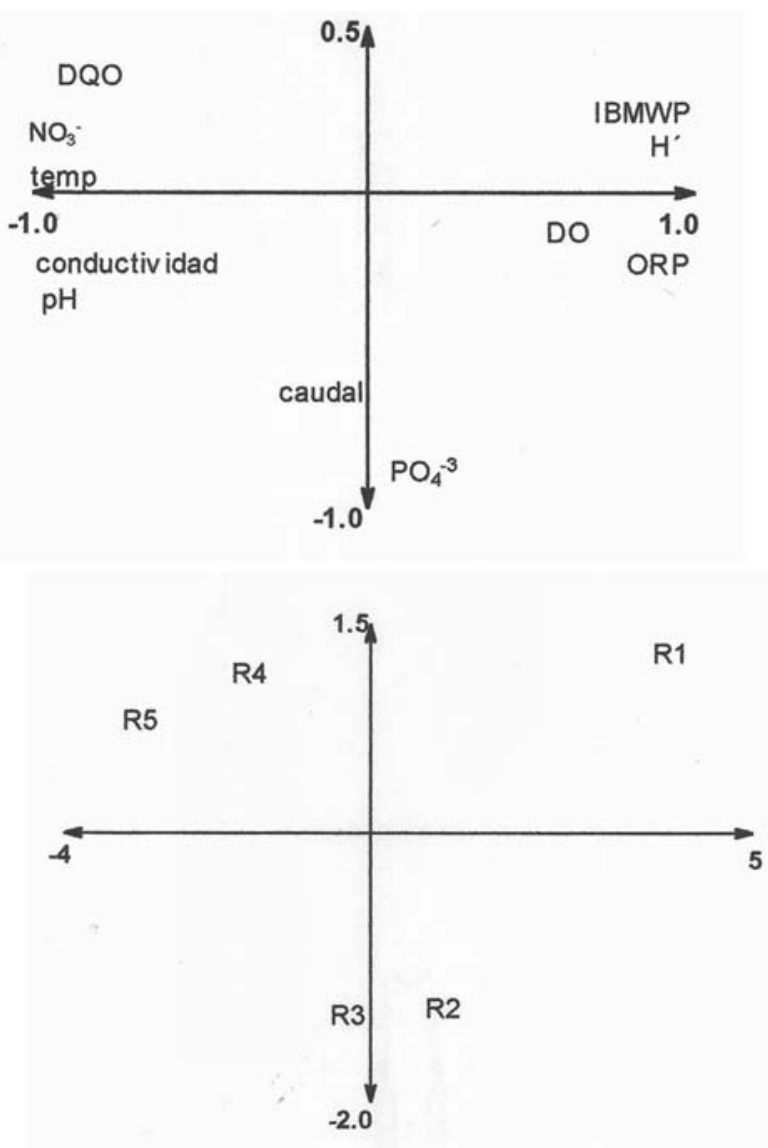

Figura 2. Proyección de las variables ambientales y las estaciones de muestreo sobre el plano definido por los ejes 1 y 2 del análisis de componentes principales. Projection of the environmental variables and sampling stations on the axis 1 and 2 of the principal components analysis plane. con 8 familias, si bien estos últimos presentaron una escasa abundancia y se localizaron casi exclusivamente en la estación de cabecera. Los efemerópteros fueron dominantes en la cabecera, mientras que el grupo oligoquetos lo fue en la estación de Ezcaray. En el resto de estaciones, los dípteros, a través de la familia Chironomidae presentaron las mayores abundancias relativas, según se aprecia en la Tabla 1.

En la Tabla 2 se presentan los valores del índice IBMWP. Se observa un estado ecológico muy bueno en la estación de cabecera. En el resto de las estaciones el estado ecológico es bueno, a excepción de la estación de Casalarreina (R5), que se encuentra en el umbral que separa las aguas clasificadas como buenas de las aceptables. Se aprecia, asimismo, una disminución del valor del índice conforme aumenta la distancia respecto a la cabecera, especialmente en Ezcaray, debido al efecto del efluente de la depuradora.

En la Tabla 3 se presentan los valores de los índices biológicos EPT riqueza y EPT/Chironomidae, y los correspondientes a la riqueza de familias, abundancia total e índice de diversidad de Shannon-Weaver. En los índices EPT riqueza y EPT/Chironomidae se observan valores bajos en las tres últimas estaciones, con dominancia del taxon Chironomidae respecto a los grupos de efemerópteros, plecópteros y tricópteros. La riqueza de familias e índice de diversidad de ShannonWeaver, presentan una tendencia a disminuir progresivamente en el sentido longitudinal del río. En el parámetro de densidad se observa, por el contrario, un aumento progresivo a lo largo del curso del río (salvo en Ezcaray).

Los valores de las variables físico-químicas para cada estación se muestran en la Tabla 4. Se observa un incremento progresivo de la concen- 

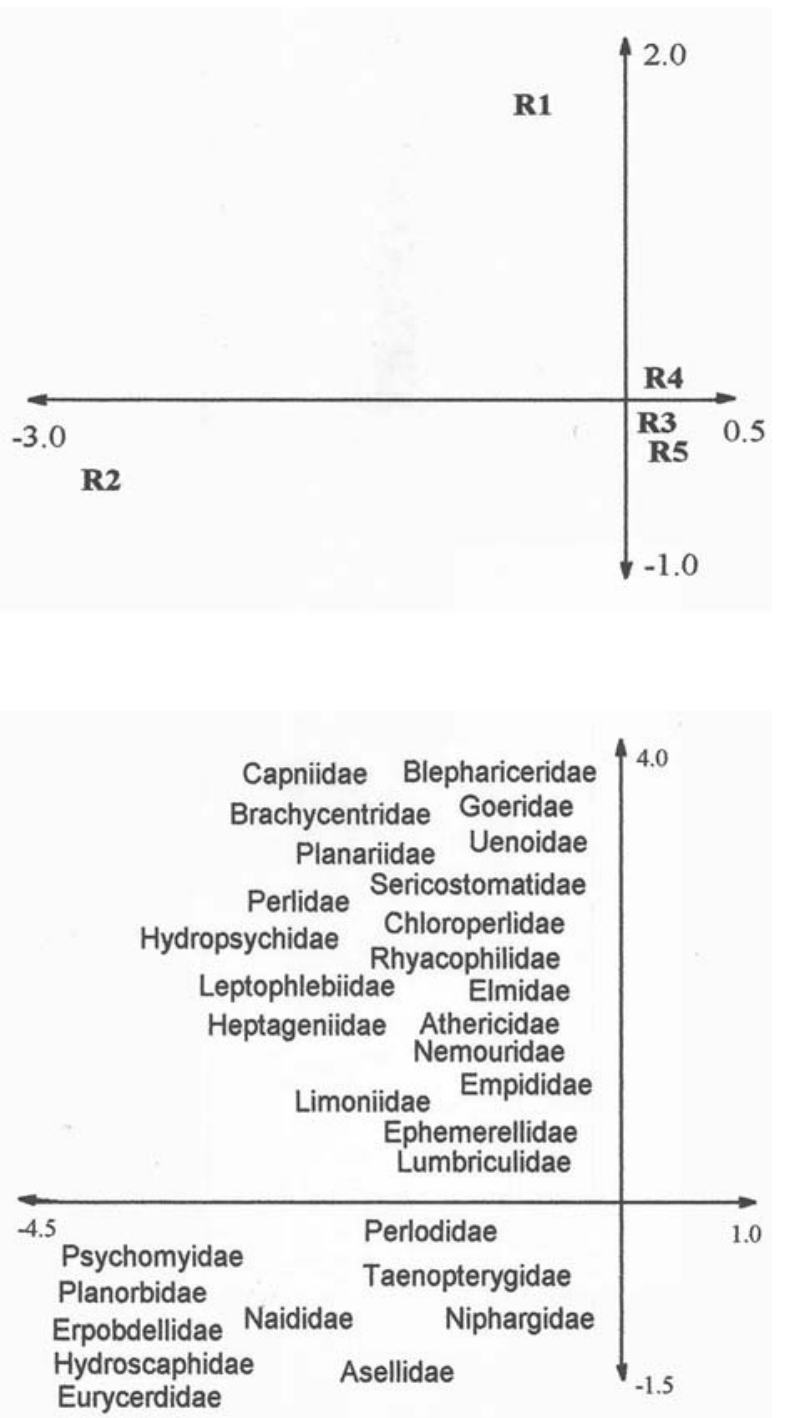

Figura 3. Diagrama de ordenación del análisis de correspondencia (ejes 1 y 2 ) de las estaciones de muestreo y las familias de macroinvertebrados. Correspondence analysis ordination diagram (axes 1 and 2) with sampling stations and macroinvertebrate families.

tración de nitrato, la DQO, la temperatura y la conductividad a lo largo del gradiente longitudinal del río. El caudal, sin embargo, manifiesta otra tendencia, presentando en Santo Domingo un valor menor a los observados en las estaciones situadas aguas arriba. En las estaciones de Ezcaray (R2) y Santurde (R3), bajo el efecto del ver- tido de efluentes residuales, se observaron altas concentraciones de ión fosfato.

En la figura 2 se muestra la proyección de las variables ambientales y las estaciones de muestreo, sobre el plano definido por los dos primeros ejes del análisis de componentes principales. Los autovalores asociados al primer y segundo eje son, respectivamente, 7.4 y 1.9 y explican en conjunto un $84.8 \%$ de la varianza. El eje 1 (67.4\% de la varianza) presenta una alta correlación negativa con el $\mathrm{pH}$, la conductividad, los nitratos, la DQO y la temperatura, y correlación positiva con el potencial redox, porcentaje de saturación de oxígeno, el índice $H^{\prime}$ y el índice IBMWP. Las estaciones de muestreo aparecen ordenadas sobre el eje 1 según su disposición en el sentido longitudinal del río, en concordancia con el gradiente de calidad. El eje $2(17.4 \%)$, muestra una correlación negativa con los fosfatos $\mathrm{y}$, en menor medida, con el caudal, y diferencia a Ezcaray y Santurde del resto de estaciones, hecho atribuible al efecto del aporte de fosfatos por la depuradora de Ezcaray.

En el análisis de correspondencias (Fig. 3) los autovalores de los dos primeros ejes $(0.432$ y 0.290$)$ explican el $82.2 \%$ de la varianza. La ordenación de las estaciones en el primer eje (49.2 \% de la varianza) separa la estación de Ezcaray (impacto del efluente de la depuradora), representada por los taxones Psychomyidae, Planorbidae, Hydroscaphidae, Eurycerdidae, Naididae y Erpobdellidae. La ordenación asociada al eje $2(32.99 \%)$ diferencia claramente la estación de cabecera del resto, hecho que refleja un cambio importante en la composición de la comunidad de macroinvertebrados asociado al gradiente longitudinal de calidad (aumento de la salinidad, DQO, temperatura y $\mathrm{pH}$; véase Fig. 2). Los taxones más representativos de este tramo de cabecera, montañoso, de aguas limpias muy poco mineralizadas y temperaturas frías, son los siguientes: Goeridae, Brachycentridae, Sericostomatidae, Uenoidae (tricópteros), Perlidae, Chloroperlidae, Capniidae (plecópteros), Blephariceridae (dípteros) y Planariidae (planarias). En el extremo negativo no se aprecia agrupaciones de taxones concretos. 
Tabla 3. EPT riqueza, EPT/ Chironomidae, riqueza de familias, densidad e índice de diversidad de Shannon-Weaver $\left(H^{\prime}\right)$ para cada estación. EPT richness, EPT/ Chironomidae, families' richness, density and Shannon-Weaver diversity index $\left(H^{\prime}\right)$ for each station.

\begin{tabular}{lcccrr}
\hline Estaciones & EPT riqueza (familias) & EPT/Chironomidae & Riqueza (familias) & Densidad (individuos/m $\mathbf{m}^{2}$ ) & $\boldsymbol{H}^{\prime}$ \\
\hline Cabecera & 24 & 1.64 & 32 & 848 & 2.37 \\
Ezcaray & 16 & 0.85 & 31 & 643 & 1.91 \\
Santurde & 9 & 0.23 & 27 & 1091 & 0.92 \\
Santo Domingo & 9 & 0.40 & 24 & 3658 & 1.11 \\
Casalarreina & 7 & 0.05 & 22 & 4301 & 0.65 \\
\hline
\end{tabular}

\section{DISCUSIÓN}

Los resultados obtenidos tras la aplicación del índice IBMWP indican que las aguas del Río Oja presentan una muy buena calidad en el tramo alto $\mathrm{y}$ una calidad buena en los tramos medio y bajo (Tabla 2). No obstante, las variaciones observadas en la composición y estructura de la comunidad de macroinvertebrados parecen evidenciar alteraciones locales en determinados puntos del sistema. En la estación de Ezcaray, el hecho de que los oligoquetos pasen a constituir el grupo taxonómico dominante, por delante de efemerópteros e incluso dípteros, puede atribuirse a la influencia del vertido de la depuradora sobre el sistema fluvial (Tabla 1). El análisis de componentes principales (Fig. 2) separa la estación de Ezcaray (mayor presencia de ortofosfato procedente de la depuradora), si bien no contamos con datos sobre otros posibles contaminantes procedentes del polígono industrial que podrían estar afectando a la estructura de la comunidad.

Los bajos valores de los índices de diversidad de Shannon, de EPT riqueza y de EPT/Chironomidae en las estaciones del tramo medio y bajo de la cuenca (Tabla 3) parecen indicar la existencia de un cierto deterioro, con claro dominio de los quironómidos sobre efemerópte- ros, plecópteros y tricópteros (comúnmente asociados a condiciones poco alteradas). La elevada proporción de quironómidos, podría también explicarse por los efectos de la crecida que tuvo lugar tres semanas antes del muestreo. Existen diversos estudios que hacen referencia al efecto destructor que este tipo de fenómenos naturales tiene sobre las comunidades de macroinvertebrados (Morais et al., 2004; Robinson et al., 2004; Rodríguez et al., 1994; Shieh \& Yang, 2000; Vitor Cortes et al., 2002; Voelz et al., 2000) debido al arrastre de los individuos, que provoca la desorganización y la disminución del tamaño de la comunidad. En estas circunstancias los taxones oportunistas y tolerantes, con un ciclo de vida corto, se ven favorecidos en las primeras fases de recolonización y recuperación. Chironomidae, Simuliidae y Baetidae son algunos de los que presentan una recuperación más rápida ante fenómenos de este tipo (Robinson et al., 2004). El escaso tiempo de recuperación transcurrido desde la crecida hasta el momento del muestreo, explicaría también los bajos valores de diversidad y riqueza detectados en los tramos medios y bajos. Pupilli \& Puig (2003) observaron en un estudio en el río Mataranya (perteneciente a la cuenca del Ebro) un descenso de más del $97 \%$ en las densidades de los taxones tras una intensa crecida. Voetz et

Tabla 4. Parámetros físico químicos en cada estación. Physico chemical-parameters at each station.

\begin{tabular}{lcccccccc}
\hline Estaciones & Temperatura $\left({ }^{\circ} \mathbf{C}\right)$ & $\begin{array}{c}\text { Caudal } \\
\left(\mathbf{m}^{\mathbf{3}} / \mathbf{s}\right)\end{array}$ & $\begin{array}{c}\text { PH } \\
\end{array}$ & $\begin{array}{c}\text { Conductividad } \\
(\boldsymbol{\mu} \mathbf{s} / \mathbf{c m})\end{array}$ & ORP $(\mathbf{m V})$ & DO $(\boldsymbol{\%})$ & $\mathbf{N O}_{\mathbf{3}}^{-}(\mathbf{m g} / \mathbf{l})$ & $\mathbf{P O}_{\mathbf{4}}^{-3}(\mathbf{m g} / \mathbf{l})$ \\
\hline Cabecera & 7.02 & 0.82 & 5.63 & 12 & 204.6 & 128.2 & 0 & 0 \\
Ezcaray & 11.88 & 1.62 & 7.22 & 109 & 197.0 & 125.0 & 1.9 & 0.3 \\
Santurde & 11.51 & 1.86 & 7.44 & 135 & 42.8 & 127.2 & 2.9 & 0.3 \\
Santo Domingo & 16.81 & 0.37 & 8.01 & 210 & 17.0 & 128.6 & 7.3 & 0 \\
Casalarreina & 15.28 & 1.72 & 7.54 & 216 & 11.8 & 102.8 & 16.9 & 0 \\
\hline
\end{tabular}


al. (2000) realizaron observaciones similares en el río Colorado, constatando que la comunidad de macroinvertebrados recuperaba los niveles de densidad y riqueza previos en un tiempo que variaba de unos meses a un año. Diversos estudios coinciden en otorgar a la comunidad de macroinvertebrados una gran capacidad de recuperación ante episodios de crecidas e inundaciones (Gibbins et al., 2001; Morais et al., 2004; Voelz et al., 2000), señalando los escasos cambios estructurales en la comunidad a una escala temporal amplia (a pesar de la recurrencia de eventos naturales de este tipo y de manifestar una cierta variabilidad a corto plazo tras los mismos). La adecuada comprensión del funcionamiento del sistema y el discernimiento entre los efectos inducidos por factores naturales y antrópicos, requieren el desarrollo de estudios a largo plazo (Naiman et al., 1995). Es necesario, por tanto, realizar campañas de muestreo en el Oja a escala estacional para profundizar en estos aspectos.

El gradiente de calidad observado en el río Oja a lo largo del perfil longitudinal parece responder a una combinación de los efectos derivados de las actividades antrópicas (actividad agrícola y vertidos de efluentes residuales) y las variaciones que de forma natural experimentan los factores ambientales del sistema. Estos factores, que parecen afectar a la distribución y estructura de la comunidad de macroinvertebrados en el Oja, son comparables a los observados por Vivas et al. (2002) en su estudio de los ríos mediterráneos de las principales cuencas españolas. A su vez, se aprecia una concordancia con las observaciones de Shiegh \& Yang (2000) en un arroyo de montaña en Taiwán situado en un área de gran actividad agrícola. Shieh \& Yang apreciaron que la aplicación de fertilizantes nitrogenados y otras sustancias químicas, explicaban los elevados valores de conductividad, concentración de nitrato y dureza detectados en el río y a su vez los bajos valores de densidad, riqueza y diversidad taxonómica obtenidos. Rodrigues Capítulo et al. (2001) también observaron en ríos de Argentina una progresiva sucesión, aguas abajo, de taxones sensibles a la contaminación por otros más tolerantes (quironómidos y oligoquetos), ante la mayor presencia de contaminantes y nutrientes procedentes de la actividad agrícola y el vertido de efluentes residuales en las estaciones de la zona baja del río. El enriquecimiento orgánico y salino moderado observado en el Oja, favorece la instalación de una comunidad más tolerante a la mineralización y carga orgánica, que contribuye a diferenciar la zona más humanizada de la zona de cabecera, como se puede apreciar en la segunda dimensión del análisis de correspondencias (Fig. 3). Los taxones característicos de aguas de cabecera (Goeridae, Brachycentridae, Sericostomatidae, Uenoidae, Blephariceridae, Perlidae, Capniidae) son sustituidos en los tramos medios y bajos por un amplio grupo de taxones más tolerantes como Chironomidae, Baetidae, Simuliidae, Gammaridae, Lumbricidae, Ceratopogonidae y Lumbriculidae.

El río presenta una buena capacidad de autodepuración, debido a las fuertes pendientes que suelen dominar la mayor parte de su perfil longitudinal, y al carácter puntual y reducido de los impactos. No obstante, debe tenerse en cuenta el posible efecto de estos impactos durante el periodo estival, especialmente, considerando que en el río Oja las variaciones de caudal asociadas a la estacionalidad natural del sistema son acentuadas, por la presión derivada de la explotación del acuífero aluvial con fines agrícolas. La menor capacidad de lavado y de dilución del sistema, y la mayor evaporación tienden a intensificar durante este periodo los efectos de la alteración antrópica y a evidenciar los procesos de contaminación (Vivas et al., 2002).

Las comunidades de los ríos están fuertemente asociadas a estas variaciones del flujo del sistema (Armitage et al., 2001; Basaguren et al., 1996; Caruso, 2002; Fleituch, 2003, Gibbins et al., 2001; Habidija et al., 2003; Wright \& Symes, 1999; Rodríguez et al., 2002). El patrón general observado en ríos mediterráneos y otros sistemas similares sometidos a variaciones estacionales, se refleje en un predominio de taxones más tolerantes a la contaminación (Morais et al., 2004; Rodriguez et al., 1994; Wood et al., 2000). Las especies reófilas y los filtradores reducen su densidad, mientras que los detritívoros la aumentan (Extence, 1981; Pearson, 1984). Así mismo, plecópteros, efemerópteros y tricópteros pierden 
importancia ante la progresiva dominancia de los grupos de odonatos, heterópteros, coléopteros y dípteros (Rieradevall et al., 1999; Vivas et al., 2003). Se ha observado, asimismo, una tendencia a la disminución de los parámetros de riqueza, densidad y abundancia de la comunidad (Caruso, 2002), si bien, existen estudios que muestran lo contrario (Morais et al., 2004; Rieradevall et al. 1999) debido a la mayor disponibilidad de alimento (alta diversidad de algas verdes filamentosas y diatomeas) y diversidad de nichos que se crean en algunos casos (Morais et al., 2004). En el río Oja algunos tramos llegan a secarse, lo cual puede intensificar los efectos sobre la comunidad macrobentónica. Algunos autores (Caruso, 2002; Delucchi, 1989) no obstante, indican que ciertos taxones han desarrollado mecanismos para permanecer durante ese periodo de gran estrés hídrico, en formas de resistencia en el sustrato. En los tramos no afectados por contaminación, como la cabecera, las variaciones de temperatura podrían explicar la ausencia de familias estenotermas que habitualmente suelen encontrarse en la zona rithron (como Heptageniidae).

Como conclusión general puede decirse que el sistema se encuentra en un buen estado de conservación, ya que los impactos son de reducida intensidad y el río presenta una buena capacidad de autodepuración, si bien merece la pena mencionar el efecto de fenómenos naturales como son las avenidas, y el posible efecto de los estiajes.

\section{AGRADECIMIENTOS}

Este trabajo de investigación se está financiando a través del Proyecto del Plan Nacional de I+D (REN2002-02550). La Unión de Agricultores y Ganaderos-Coordinadora de Agricultores y Ganaderos (UAGR- COAG) apoya el desarrollo de la investigación mediante un Contrato Tecnológico (ref. 2003/672).

La Consejería de Educación de la Comunidad de Madrid y el Fondo Social Europeo (F.S.E.) participan mediante la financiación de una beca de Formación de Personal Investigador.

\section{BIBLIOGRAFÍA}

ALBA-TERCEDOR, J. \& A. SÁNCHEZ-ORTEGA. 1988. Un método rápido y simple para evaluar la calidad biológica de las aguas corrientes basado en el de Hellawell (1978). Limnetica, 4: 51-56.

ALBA-TERCEDOR, J. 1996. Macroinvertebrados acuáticos y calidad de las aguas de los ríos. IV Simposio del Agua en Andalucía (SIAGA), Almería, España: 203-213.

ALBA-TERCEDOR, J., P. JÁIMEZ-CÚELLAR, M. ALVAREZ, J. AVILÉS, N. BONADA, J. CASAS, A. MELLADO, M. ORTEGA, I. PARDO, N. PRAT, M. RIERADEVALL, S. ROBLES, C. E. SÁINZ-CANTERO, A. SÁNCHEZ-ORTEGA, M. L. SÚAREZ, M. TORO, M. R. VIDAL-ABARCA, S. VIVAS \& C. ZAMORA-MUÑOZ. 2002. Caracterización del estado ecológico de ríos mediterráneos ibéricos mediante el índice IBMWP (antes BMWP'). Limnetica, 21: 175-185.

APHA. 1998. Standard methods for the examination of water and wastewater, $20^{\text {th }}$ ed. American Public Health Association, American Water Works Association \& Water Environment Federation. USA. 1134 pp.

ARMITAGE P. D., D. MOSS, J. F. WRIGHT \& M. T. FURSE. 1983. The performance of a new biological water quality score system based on macroinvertebrates over a wide range of unpolluted running-water sites. Wat. Res., 17: 333-347.

ARMITAGE, P. D., K. LATTMANN, N. KNEEBONE \& I. HARRIS. 2001. Bank profile and structure as determinants of macroinvertebrate assemblagesseasonal changes and management. Regul. Rivers: Res. Mgmt., 17: 543-556.

BARBOUR, M. T., J. GERRITSEN, B. D. SNYDER \& J. B. STRIBLING. 1999. Rapid Bioassessment Protocols or Use in Streams and Wadeable Rivers: Periphyton, Benthic Macroinvertebrates and Fish. U.S. Environmental Protection Agency, EPA-891B99-002. Washington D.C. 339 pp.

BASAGUREN, A., A. ELOSEGUI, J. POZO. 1996. Changes in the trophic structure of benthic macroinvertebrate communities associated with food availability and stream flow variations. Internationale Reveu Der Gesamten Hydrobiologie, 81: 79-91.

CARUSO, B.S. 2002. Temporal and spatial patterns of extreme low flows and effects on stream ecosystems in Otago, New Zealand. J. Hydrol., 257: 115-133.

COIMBRA, C. N., M. A. S. GRAÇA \& R. M. CORTES. 1996. The effects of a basic effluent on ma- 
croinvertebrate community structure in a temporary Mediterranean river. Env. Poll., 94: 301-307.

DELUCHI, C. M. 1989. Movement patterns of invertebrates in temporary and permanent streams. Oecologia, 78: 199-207.

EXTENCE C. A. 1981. The effect of drought on benthic invertebrate communities in a lowland river. Hydrobiologia, 83: 217-224.

FLEITUCH, T. 2003. Structure and Functional Organization of Benthic Invertebrates in a Regulated Stream. Internat. Rev. Hydrobiol., 88: 332-344

GIBBINS, C. N., C. F. DILKS, R. MALCOLM, C. SOULSBY \& S. JUGGINS. 2001. Invertebrate communities and hydrological variation in Cairngorm mountain streams. Hydrobiologia, 462: 205-219.

GUEROLD, F. 2000. Influence of taxonomic determination level on several community indices. Wat. Res., 34: 487-492.

HABIDIJA I., I. RADANOVIC, B. PRIMC-HABDIJA, R. MATONICKIN, M. KUCINIC. 2003. River discharge regime as a factor affecting the changes in community and functional feeding group composition of macroinvertebrates on a cobble substrate in the Sava river. Biologia, 58: 217-229.

INSTITUTO GEOLÓGICO Y MINERO DE ESPAÑA, 1985. Plano Hidrogeológico $\mathrm{n}^{\mathrm{o}} 1$, Investigación hidrogeológica del cuaternario del río Glera (La Rioja). Clave: ZP-1490. Escala 1:50.000.

MORAIS, M., P. PINTO, P. GUILHERME, J. ROSADO \& I. ANTUNES. 2004. Assessment of temporary streams: the robustness of metric and multimetric indices under different hydrological conditions. Hydrobiologia, 516: 229-249.

NAIMAN, R. J., J. J. MAGNUSON, D. M. MCKNIGHT \& J. A. STANFORD. 1995. The Freshwater Imperative. Island Press. Washington, D.C. 165 pp.

PEARSON, R. G. 1984. Temporal changes in the composition and abundance of the macroinvertebrate communities of the River Hull. Arch. Hydrobiol., 100: 273-298

PUPILLI, E. \& M. A. PUIG. 2003. Effects of a major flood on the mayfly and stonefly populations in a Mediterranean stream (Matarranya Stream, Ebro River basin, North East of Spain). Research Update on Ephemeroptera \& Plecoptera. E. Gaino (ed.): 381-389. University of Perugia, Perugia.

RICO, E., A. RALLO, M. A. SEVILLANO \& M. I. ARRETXE. 1992. Comparison of several biological indices based on river macroinvertebrate benthic community for assessment of running water quality. Ann. Limnol., 28: 147-156.
RIERADEVALL, M., N. BONADA, N. PRAT. 1999. Community structure and water quality in the Mediterranean streams of a natural park (St. Llorenc del Munt, NE Spain). Limnetica, 17: 45-56.

ROBINSON, C. T., U. R. S. UEHLINGER \& M. T. MONAGHAN. 2004. Stream ecosystem response to multiple experimental floods from a reservoir. River Res. Applic., 20: 359-377.

RODRÍGUEZ, S. E., C. FERNÁNDEZ-ALÁEZ, M. FERNÁNDEZ-ALÁEZ \& E. BÉCARES. 1994. Caracterización de la comunidad de macroinvertebrados de la cuenca alta del río Cares. Limnetica, 10: 93-100.

RODRIGUES CAPÍTULO, A., M. TANGORRA \& C. OCÓN. 2001. Use of benthic macroinvertebrates to assess the biological status of Pampean streams in Argentina. Aquatic Ecology, 35: 109-119.

SHANNON, C. E. \& W. WEAVER. 1949. The Mathematical Theory of Communication. Univ. of Illinois Press. Urbana. 119 pp.

SHIEH, S. \& YANG, P. 2000. Community Structure and functional Organization of Aquatic Insects in an Agricultural Mountain Stream of Taiwan: 1985-1986 and 1995-1996. Zoological Studies, 39: 191-202.

STATSOFT, INC. 2003. Statistica for Windows. Tulsa, Oklahoma.

TACHET, H., P. RICHOUX, M. BOURNAUD \& P. USSEGLIO-POLATERA. 2002. Invertébrés d'eau douce. Systématique, biologie, écologie. CNRS ed. París.

VALLADOLID, M., J. J. MARTINEZ-BASTIDA, M. ARAUZO \& C. GUTIERREZ. 2006. Abundancia y biodiversidad de los macroinvertebrados del río Oja (La Rioja, España). Limnetica, 25: 133-140.

VIVAS, S., J. CASAS, I. PARDO, S. ROBLES, N. BONADA, A. MELLADO, N. PRAT, J. ALBATERCEDOR, M. ALVAREZ, M. R. VIDAL.ABARCA, C. ZAMORA-MUÑOZ \& G. MOYÁ. 2002. Aproximación multivariante en la exploración de la tolerancia ambiental de las familias de macroinvertebrados de los ríos mediterráneos del proyecto GUADALMED. Limnetica, 21: 149-173.

VITOR CORTES, R. M., M. T. FERREIRA, S. V. OLIVEIRA \& D. OLIVEIRA. 2002. Macroinvertebrate community structure in a regulated river segment with different flow conditions. River Res. Applic., 18: 367-382.

VOELZ, N. J., S. H. SHIEH \& J. V. WARD. 2000. Long-term monitoring of benthic macroinvertebrate community structure: a perspective from a Colorado river. Aquatic Ecology, 34: 261-278. 
WOOD, P. G., M. D. AGNEW Y G. E. PETTS. 2000. Flow variations and macroinvertebrate community responses in a small groundwater-dominated stream in south-eastEngland. Hydrol. Process., 14:3133-3147.

WRIGHT, J. F. \& K. L. SYMES. 1999. A nine-year study of the macroinvertebrate fauna of a chalk stream. Hydrol. Process., 13: 371-385.

ZALDÍVAR, C. 1994. Atlas de distribución de los peces de la Comunidad Autónoma de La Rioja. Zubia Monográfico, 6: 71-102. 
